\title{
Global Existence and Decay Estimates of Energy of Solutions for a New Class of $p$-Laplacian Heat Equations with Logarithmic Nonlinearity
}

\author{
Salah Mahmoud Boulaaras $\mathbb{D}^{1,},{ }^{1,2}$ Abdelbaki Choucha, ${ }^{3}$ Abderrahmane Zara, ${ }^{4}$ \\ Mohamed Abdalla, ${ }^{5,6}$ and Bahri-Belkacem Cheri $\mathbb{D}^{1,7}$ \\ ${ }^{1}$ Department of Mathematics, College of Sciences and Arts, ArRas, Qassim University, Saudi Arabia \\ ${ }^{2}$ Laboratory of Fundamental and Applied Mathematics of Oran (LMFAO), University of Oran 1, Oran, 31000 Oran, Algeria \\ ${ }^{3}$ Laboratory of Operator Theory and PDEs: Foundations and Applications, Department of Mathematics, Faculty of Exact Sciences, \\ University of El Oued, El Oued, Algeria \\ ${ }^{4}$ Department of Mathematics, Informatics and Systems (LAMIS), Larbi Tebessi University, 12002 Tebessa, Algeria \\ ${ }^{5}$ Mathematics Department, College of Science, King Khalid University, Abha 61413, Saudi Arabia \\ ${ }^{6}$ Mathematics Department, Faculty of Science, South Valley University, Qena 83523, Egypt \\ ${ }^{7}$ Preparatory Institute for Engineering Studies in Sfax, Tunisia
}

Correspondence should be addressed to Bahri-Belkacem Cheri; bahi1968@yahoo.com

Received 3 February 2021; Revised 24 February 2021; Accepted 3 March 2021; Published 16 March 2021

Academic Editor: Chuanjun Chen

Copyright (C) 2021 Salah Mahmoud Boulaaras et al. This is an open access article distributed under the Creative Commons Attribution License, which permits unrestricted use, distribution, and reproduction in any medium, provided the original work is properly cited.

\begin{abstract}
The present research paper is related to the analytical studies of $p$-Laplacian heat equations with respect to logarithmic nonlinearity in the source terms, where by using an efficient technique and according to some sufficient conditions, we get the global existence and decay estimates of solutions.
\end{abstract}

\section{A Brief History and Contribution}

Consider the following nonlinear $p$-Laplacian problem: equation with logarithmic nonlinearity:

$$
\left\{\begin{array}{l}
u_{t}-\operatorname{div}\left(|\nabla u|^{p-2} \nabla u\right)+|u|^{p-2} u=|u|^{p-2} u \ln |u|, x \in \Omega, t>0 \\
u(x, 0)=u_{0}(x), x \in \Omega \\
u(x, t)=0, x \in \partial \Omega, t \geq 0
\end{array}\right.
$$

where $\Omega \subset R^{n}$ is a bounded domain with smooth boundary $\partial \Omega$, the function $u_{0}$ is given initial data and exponent $p$ verify

$$
\left\{\begin{array}{l}
2<p<\infty, \quad \text { if } n \leq p, \\
2<p<\frac{n p}{n-p}, \quad \text { if } n>p .
\end{array}\right.
$$

In the last few decades, the researchers have shown significant interest in polynomial nonlinear terms in different areas, such as edge detection, viscoelasticity, engineering, electromagnetic, electrochemistry, cosmology, signal processing material science, turbulence, diffusion, physics, and acoustics. Many other problems in applied sciences are also modeled by linear and nonlinear evolutionary partial differential equations [1-13]. Various dynamical systems in physics and engineering are also modeled by using evolutionary differential equations. Many researchers have contributed a lot to provide an outstanding history of the evolutionary differential partial equations related to $p(x)$-Laplacian such as [13-17].

The majority of problems in science are nonlinear, and it is not easy to find its analytical solutions. The physical problems are mostly designed by using higher nonlinear partial differential equations (PDEs). It is found to be very difficult to find the exact or analytical solutions for such problems. 
However, in the last several centuries, many scientists have made significant progress and adopted different techniques to study the analytical side of the nonlinear PDEs. Through recent years and in the literature on nonlinear PDEs, logarithmic nonlinearity has received much interest from mathematicians and physicists. If we read in recent research, we notice that logarithmic nonlinearity has been entered into nonrelativistic wave equations that describe spinning particles that move in an external electromagnetic field and in the relativistic wave equation for spinless particles (see, for example, $[2,4,18,19])$. In addition to what we mentioned above, this type of nonlinearity is used in various branches of physics such as optics, nuclear physics, geophysics, and inflationary cosmology (to read about this in detail, see [18-31]). Given all the basic previous meanings in physics, the study of universal solutions of this type of nonlinear logarithms is of great interest on the part of mathematicians.

Recently, Wu and Xue in [32] gave the uniformly proof of energy decay of the solution using the multiplier method of the following problem:

$$
u_{t t}-\operatorname{div}\left(|\nabla u|^{p-2} \nabla u\right)-\Delta u_{t}+\left|u_{t}\right|^{q-1} u_{t}=|u|^{p-1} u
$$

Moreover, the author in [33] studied the exponential and polynomial decay rate of solutions for seminar problem (3) by applying the inequality of Nakao.

On the another handle, for a Laplacian parabolic equation related to the logarithmic in the right-hand side, the authors in [24] gave the analytical side of the following problem:

$$
u_{t}-\Delta u-\Delta u_{t}=u \ln u
$$

Then, in [27], Nhan and Truong studied the global existence, decay together with the blow up the solutions of the following problem:

$$
u_{t}-\operatorname{div}\left(|\nabla u|^{p-2} \nabla u\right)-\Delta u_{t}=|u|^{p-2} u \ln |u|
$$

where $p>2$. In addition, in [25], Cao and Liu gave for $1<p$ $<2$, the blow up and global boundedness results of problem (5).

Most recently, in [14], Piskin et al. studied the $p$-Laplacian hyperbolic case

$u_{t t}-\operatorname{div}\left(|\nabla u|^{p-2} \nabla u\right)+|u|^{p-2} u+u_{t}=|u|^{p-2} u \ln |u|, x \in \Omega, t>0$.

Motivated by the last mentioned papers, especially [14], in this current research, we consider problem (1) with the presence of nonlinear diffusion $\Delta_{p}=\operatorname{div}\left(|\nabla u|^{p-2} \nabla u\right)$, logarithmic nonlinearity $|u|^{p-2} u \ln |u|$ together with a damping term which is an extension of the previous recent analytical study in [14], where the authors considered the hyperbolic case without damping terms. Our goal is to exploit a potential well method for problem (1) in order to obtain global existence and decay estimate of solutions. More precisely, we give the global existence and decay estimates of solutions under some sufficient conditions.

\section{Preliminaries}

In this section, we put the definitions and lemmas that we need in the rest of the paper:

$$
\|u\|_{p}=\|u\|_{L^{p}(\Omega)},\|u\|_{1, s}=\|u\|_{W_{0}^{1, p}(\Omega)}=\left(\|u\|_{p}+\|\nabla u\|_{p}\right)^{1 / p}
$$

for $1<p<\infty$. We denote the positive constants by $C$ and $C_{i}$ $(i=1,2, \cdots)$.

We give the function of energy by

$$
E(t)=\frac{1}{p}\|\nabla u\|_{p}^{p}+\frac{1}{p}\|u\|_{p}^{p}-\frac{1}{p} \int_{\Omega} \ln |u| u^{p} d x+\frac{1}{p^{2}}\|u\|_{p}^{p} .
$$

Lemma 1. $E(t)$ is a nonincreasing function, for $t \geq 0$

$$
E^{\prime}(t)=-\left\|u_{t}\right\|^{2} \leq 0
$$

Proof. Multiplying equation (1) by $u_{t}$ and using the integration on $\Omega$, we have

$$
\begin{gathered}
-\int_{\Omega} \operatorname{div}\left(|\nabla u|^{p-2} \nabla u\right) u_{t} d x+\int_{\Omega}|u|^{p-2} u u_{t} d x+\int_{\Omega} u_{t} u_{t} d x \\
\quad=\int_{\Omega} u^{p-2} u \ln |u| u_{t} d x
\end{gathered}
$$

$\frac{d}{d t}\left(\frac{1}{p}\|\nabla u\|_{p}^{p}+\frac{1}{p}\|u\|_{p}^{p}-\frac{1}{p} \int_{\Omega} \ln |u| u^{p} d x+\frac{1}{p^{2}}\|u\|_{p}^{p}\right)=-\left\|u_{t}\right\|^{2}$.

Thus,

$$
E^{\prime}(t)=-\left\|u_{t}\right\|^{2}
$$

Lemma 2 (see $[5,14])$. Let $u$ be any function $u \in W_{0}^{1, p}\left(R^{n}\right) \backslash$ $\{0\}$. Then, for $p>1, \mu>0$

$$
\begin{aligned}
& p \int_{R^{n}} u^{p} \ln \left(\frac{|u|}{\|u\|_{L^{p}\left(R^{n}\right)}}\right) d x \\
& \quad \leq \mu \int_{R^{n}}|\nabla u|^{p} d x-\frac{n}{p} \ln \left(\frac{p \mu e}{n \mathscr{L}_{p}}\right) \int_{R^{n}}|u|^{p} d x,
\end{aligned}
$$

where

$$
\mathscr{L}_{p}=\frac{p}{n}\left(\frac{p-1}{e}\right)^{p-1} \pi^{-p / 2}\left[\frac{\Gamma(n / 2+1)}{\Gamma(n(p-1) / p+1)}\right]^{p / n} .
$$


Remark 3. Let $u \in W_{0}^{1, p}(\Omega) \backslash\{0\}$ and by defining $u(x)=0$ for $x \in R^{n} \backslash \Omega$, we can write

$$
\begin{aligned}
& p \int_{\Omega} u^{p} \ln \left(\frac{|u|}{\|u\|_{L^{p}(\Omega)}}\right) d x \\
& \quad \leq \mu \int_{\Omega}|\nabla u|^{p} d x-\frac{n}{p} \ln \left(\frac{p \mu e}{n \mathscr{L}_{p}}\right) \int_{\Omega}|u|^{p} d x
\end{aligned}
$$

Lemma 4 (see [27]). Let $\vartheta>0$. Therefore, we can easy give the following result:

$$
\log s \leq C s^{\vartheta}
$$

$\forall s \in[1, \infty)$, such as $C=e^{-1} / \vartheta$.

Remark 5. According to Lemma 4, we have

$$
s^{p} \log s \leq C s^{p+\vartheta}, s \in[1, \infty) .
$$

Lemma 6 (see [34]).

(i) For all function $u \in W_{0}^{1, p}(\Omega)$, we have

$$
\|u\|_{q} \leq B_{q, p}\|\nabla u\|_{p}
$$

for every $q \in[1, \infty]$ if $n \leq p$, and $1 \leq q \leq n p /(n-p)$ if $n>p$. We choose constant $B_{q, p}$ related only on $\Omega$, p and $q$. Denote $B_{p, p}$ by $B_{p}$.

(ii) For every $u \in W_{0}^{1, p}(\Omega), p \geq 1$ with $r \geq 1$, we get

$$
\|u\|_{q} \leq C\|\nabla u\|_{p}^{\alpha}\|u\|_{r}^{1-\alpha}
$$

where $C>0$,

$$
\alpha=\left(\frac{1}{r}-\frac{1}{q}\right)\left(\frac{1}{n}-\frac{1}{p}+\frac{1}{r}\right)^{-1}
$$

and we have the following:

(i) For $p \geq n=1, r \leq q \leq \infty$

(ii) For $n>1$ and $p<n, q \in[r,(n p / n-p)]$ if $r \leq n p / n-p$ and $q \in[r,(n p / n-p)]$ if $r \leq n p / n-p$

(iii) For $p=n>1, r \leq q<\infty$

(iv) For $p>n>1, r \leq q \leq \infty$

\section{Result of the Global Existence}

We give in this section the proof of the global existence for (1). First, putting the following functionals:

$$
\begin{gathered}
J(u)=\frac{1}{p}\|\nabla u\|_{p}^{p}+\frac{p+1}{p^{2}}\|u\|_{p}^{p}-\frac{1}{p} \int_{\Omega} \ln |u| u^{p} d x \\
I(u)=\|\nabla u\|_{p}^{p}+\|u\|_{p}^{p}-\int_{\Omega} \ln |u| u^{p} d x
\end{gathered}
$$

Hence, (21) and (22) give

$$
J(u)=\frac{1}{p} I(u)+\frac{1}{p^{2}}\|u\|_{p}^{p},
$$

and we have

$$
E(u)=J(u)
$$

As in [35], the potential depth of the well is given as

$$
0<d=\inf _{u}\left\{\sup _{\lambda \geq 0} J(\lambda u): u \in W_{0}^{1, p}(\Omega),\|u\|_{p}^{p} \neq 0\right\}
$$

$$
0<d=\inf _{u \in \aleph} J(t)
$$

Hence, two sets can be assigned, the first stable $W$ and the second $V$ unstable by

$$
\begin{gathered}
W=\left\{u \in W_{0}^{1, p}(\Omega): J(u)<d, I(u)>0\right\} \cup\{0\}, \\
V=\left\{u \in W_{0}^{1, p}(\Omega): J(u)<d, I(u)<0\right\} .
\end{gathered}
$$

Lemma 7. Let $u$ be all function $u \in W_{0}^{1, p}(\Omega) \backslash\{0\},\|u\|_{p}^{p} \neq 0$ and let $(\lambda)=J(\lambda u)$. Hence, we have

(i) $\lim _{\lambda \rightarrow \infty} g(\lambda)=-\infty, \lim _{\lambda \rightarrow 0^{+}} g(\lambda)=0$

(ii) $I(\lambda u)=\lambda g^{\prime}(\lambda)\left\{\begin{array}{l}>0,0 \leq \lambda<\lambda^{*}, \\ =0, \lambda=\lambda^{*}, \\ <0, \lambda<\lambda^{*}<\infty\end{array}\right.$

where

$$
\lambda^{*}=\exp \left(\frac{\|\nabla u\|_{p}^{p}+\|u\|_{p}^{p}-\int_{\Omega} \ln |u \| u|^{p} d x}{\|u\|_{p}^{p}}\right) .
$$

Proof.

(i) From $g(\lambda)$ which we get 


$$
\begin{aligned}
g(\lambda)=J(\lambda u)= & \frac{1}{p}\|\lambda \nabla u\|_{p}^{p}+\frac{p+1}{p^{2}}\|\lambda u\|_{p}^{p} \\
& -\frac{1}{p} \int_{\Omega} \ln |\lambda u|(\lambda u)^{p} d x=\frac{\lambda^{p}}{p}\|\nabla u\|_{p}^{p} \\
& +\frac{\lambda^{p}}{p}\left(\frac{p+1}{p}-\ln |\lambda|\right)\|u\|_{p}^{p} \\
& -\frac{\lambda^{p}}{p} \int_{\Omega} \ln |u \| u|^{p} d x .
\end{aligned}
$$

According to $\|u\|_{p}^{p} \neq 0$, we find $\lim _{\lambda \rightarrow \infty} g(\lambda)=-\infty$, and $\lim _{\lambda \rightarrow 0}$ $g(\lambda)=0$.

(ii) From the derivative of $g(\lambda)$, we get

$$
\begin{aligned}
g^{\prime}(\lambda)= & \frac{d}{d \lambda} J(\lambda u) \\
= & \lambda^{p-1}\left(\|\nabla u\|_{p}^{p}+(1-\ln |\lambda|)\|u\|_{p}^{p}\right. \\
& \left.-\int_{\Omega} \ln |u||u|^{p} d x\right) .
\end{aligned}
$$
ing

There exists a unique $\lambda^{*}$ verify $\left.(d / d \lambda) J(\lambda u)\right|_{\lambda=\lambda^{*}}$, by tak-

$$
\lambda^{*}=\exp \left(\frac{\|\nabla u\|_{p}^{p}+\|u\|_{p}^{p}-\int_{\Omega} \ln |u \| u|^{p} d x}{\|u\|_{p}^{p}}\right) .
$$

Of course, we note that the recent property is the result of the following:

$$
\lambda \frac{d J(\lambda u)}{d \lambda}=\lambda g^{\prime}(\lambda)=I(\lambda u) .
$$

Thus, we have the desired results such that

$$
I(\lambda u)=\lambda g^{\prime}(\lambda) \begin{cases}>0, & 0 \leq \lambda<\lambda^{*} \\ =0, & \lambda=\lambda^{*} \\ <0, & \lambda<\lambda^{*}<\infty\end{cases}
$$

Lemma 8. For every $u \in W_{0}^{1, p}(\Omega) \backslash\{0\}$ and $l=e^{\left(n+p^{2}\right) / p^{2}}$ $\left(p^{2} / n \mathscr{L}_{p}\right)^{n / p^{2}}$, we get

(i) If $0<\|u\|_{p}<l$, then $I(u)>0$

(ii) If I $(u)<0$, then $\|u\|_{p}>l$

(iii) If $I(u)=0$, then $\|u\|_{p} \geq l$
Proof. According to inequality of logarithmic Sobolev, it can be found

$$
\begin{aligned}
I(u)= & \|\nabla u\|_{p}^{p}+\|u\|_{p}^{p}-\int_{\Omega}\left(\ln \frac{|u|}{\|u\|_{p}}+\ln \|u\|_{p}\right)|u|^{p} d x \\
\geq & \|\nabla u\|_{p}^{p}+\left(1-\ln \|u\|_{p}\right)\|u\|_{p}^{p} \\
& -\left[\frac{\mu}{p} \int_{\Omega}|\nabla u|^{p} d x-\frac{n}{p^{2}} \ln \left(\frac{p \mu e}{n \mathscr{L}_{p}}\right) \int_{\Omega}|u|^{p} d x\right] \\
\geq & \left(1-\frac{\mu}{p}\right)\|\nabla u\|_{p}^{p}+\left(1-\ln \|u\|_{p}+\frac{n}{p^{2}} \ln \left(\frac{p \mu e}{n \mathscr{L}_{p}}\right)\right)\|u\|_{p}^{p} .
\end{aligned}
$$

Selecting $\mu=p$ in (34) gives

$$
I(u) \geq\left(1-\ln \|u\|_{p}+\frac{n}{p^{2}} \ln \left(\frac{p^{2} e}{n \mathscr{L}_{p}}\right)\right)\|u\|_{p}^{p} .
$$

Thus, we have

(i) If $0<\|u\|_{p}<l$, then $I(u)>0$ using the last inequality

(ii) Suppose that $I(u)<0$. This is due to (35), and it

$$
\|u\|_{p} \geq e^{\left(n+p^{2}\right) / p^{2}}\left(\frac{p^{2}}{n \mathscr{L}_{p}}\right)^{n / p^{2}}=l
$$

(iii) Similar to the proof of (ii), we prove (iii)

As for functional $J$, it represents the Nehari manifold

$$
\aleph=\left\{u \in W_{0}^{1, p}(\Omega) \backslash\{0\}: I(u)=0\right\} .
$$

Using Lemma 7 in order to prove that $\aleph$ is an unempty set, consider that if $u \in \aleph$, we obtain

$$
J(u)=\frac{1}{p^{2}}\|u\|_{p}^{p}
$$

We use (23). Further, it proves that $J$ is coercive with respect to $\aleph$. In addition, if we give $\Omega_{1}$ and $\Omega_{2}$ such that

$$
\begin{aligned}
& \Omega_{1}=\{x \in \Omega:|u(x)<1|\}, \\
& \Omega_{2}=\{x \in \Omega:|u(x) \geq 1|\} .
\end{aligned}
$$


From Remark 5, we can get that

$$
\begin{aligned}
\int_{\Omega}|u|^{p} \ln |u| d x & \leq \int_{\Omega_{1}}|u|^{p} \ln |u| d x+\int_{\Omega_{2}}|u|^{p} \ln |u| d x \\
& \leq C \int_{\Omega_{2}}|u|^{p+\zeta} d x \leq C\|u\|_{p+\zeta}^{p+\zeta}
\end{aligned}
$$

where $\zeta>0$. Under Lemma 6 , we get

$$
\int_{\Omega} \ln \left|u\left\|\left.u\right|^{p} d x \leq C\right\| u\left\|_{p+\zeta}^{p+\zeta} \leq C\right\| \nabla u\left\|_{p}^{\alpha(p+\zeta)}\right\| u \|_{p}^{(1-\alpha)(p+\zeta)}\right.
$$

where

$$
\alpha=\left(\frac{1}{p}-\frac{1}{p+\zeta}\right)\left(\frac{1}{n}-\frac{1}{p}+\frac{1}{p}\right)^{-1}=\frac{n \zeta}{p(p+\zeta)}
$$

Choosing $\zeta<p^{2} / n$, we obtain

$$
\alpha(p+\zeta)<p
$$

By using Young's inequality together with (41), we get

$$
\int_{\Omega}|u|^{p} \ln |u| d x \leq \varepsilon\|\nabla u\|_{p}^{p}+C_{\varepsilon}\left(\|u\|_{p}^{p}\right)^{\beta}
$$

where $\varepsilon>0$ and $\beta=(1-\alpha)(p+\zeta) / p-\alpha(p+\zeta)>1$. As $u \in \aleph$, by (22) and (44), we get

$$
\begin{gathered}
\|u\|_{p}^{p}+\|\nabla u\|_{p}^{p}=\int_{\Omega}|u|^{p} \ln |u| d x, \\
\|u\|_{p}^{p}+\|\nabla u\|_{p}^{p} \leq \varepsilon\|\nabla u\|_{p}^{p}+C_{\varepsilon}\left(\|u\|_{p}^{p}\right)^{\beta}, \\
\|\nabla u\|_{p}^{p} \leq \varepsilon\|\nabla u\|_{p}^{p}+C_{\varepsilon}\left(\|u\|_{p}^{p}\right)^{\beta}, \\
(1-\varepsilon)\|\nabla u\|_{p}^{p} \leq C_{\varepsilon}\left(\|u\|_{p}^{p}\right)^{\beta} .
\end{gathered}
$$

Select $\varepsilon<1$. Then, combining (38) and (44), we find

$$
J(t)=\frac{1}{p^{2}}\|u\|_{p}^{p} \geq C_{\varepsilon}\left(\|\nabla u\|_{p}^{p}\right)^{1 / \beta}
$$

Hence, the coercivity of $J$ on $\aleph$.

\section{Lemma 9.}

(i) The depth of the potential well is given by

$$
d=\inf _{u \in \aleph} J(u)=\inf \left\{\sup _{\lambda \geq 0} J(\lambda u): u \in W_{0}^{1, p}(\Omega) /\{0\},\|u\|_{p}^{p} \neq 0\right\}
$$

(ii) $d$ admis a positive lower bound, given by

$$
d \geq \frac{1}{p^{2}} e^{\left(n+p^{2}\right) / p}\left(\frac{p^{2}}{n \mathscr{L}_{p}}\right)^{n / p}=\frac{l^{p}}{p^{2}}=K,
$$

where $\mathscr{L}_{p}$ is given as in Lemma 2

(iii) There exists a positive function $u \in \aleph$, verify $J(u)=d$

Proof.

(i) According to Lemma 7, it implies that for every $u \in$ $W_{0}^{1, p}(\Omega) \backslash\{0\}$, there exists a $\lambda^{*}$, verify $I\left(\lambda^{*} u\right)=0$, that is $\lambda^{*} u \in \aleph$. Using (47) gives

$$
J\left(\lambda^{*} u\right) \geq \inf _{u \in \aleph} J(u)=d
$$

From Lemma 7 , the maximizer of $J(\lambda u)$ is exact $\lambda^{*}$, such that

$$
\sup _{\lambda \geq 0} J(\lambda u)=J\left(\lambda^{*} u\right)=\frac{1}{p} I\left(\lambda^{*} u\right)+\frac{1}{p^{2}}\left\|\lambda^{*} u\right\|_{p}^{p}=\frac{1}{p^{2}}\left\|\lambda^{*} u\right\|_{p}^{p}
$$

By the combination of (50) and (49), we find

$$
\inf _{u \in W_{0}^{1, p}(\Omega) /\{0\}} \sup _{\lambda \geq 0} J(\lambda u)=\inf _{u \in W_{0}^{1, p}(\Omega) /\{0\}} J\left(\lambda^{*} u\right) \geq d .
$$

So that, as $u \in W_{0}^{1, p}(\Omega) \backslash\{0\}$, we have $d \neq 0$. And if $u \in \aleph$ by (30), we obtain that $\lambda^{*}$ is the only critical point in $(0, \infty)$ of the mapping $g(\lambda)$. Therefore,

$$
\sup _{\lambda>0} J(\lambda u)=J(u)
$$

for any $u \in \aleph$. Then,

$$
\inf _{u \in W_{0}^{1, p}(\Omega) /\{0\}} \sup _{\lambda>0} J(\lambda u) \leq \inf _{u \in \aleph} \sup _{\lambda>0} J(\lambda u)=\inf _{u \in \aleph} J(u)=d .
$$

By (51) and (53), (i) is obtained.

(ii) From Lemma 7, $\forall u \in W_{0}^{1, p}(\Omega) \backslash\{0\}$, we get $I\left(\lambda^{*} t\right)$ $=0$. Lemma 8 gives 


$$
\left\|\lambda^{*} u\right\|_{p} \geq e^{\left(n+p^{2}\right) / p^{2}}\left(\frac{p^{2}}{n \mathscr{L}_{p}}\right)^{n / p^{2}}=l
$$

By using (50) and (54), we get

$$
\sup _{\lambda>0} J(\lambda u) \geq \frac{l^{p}}{p^{2}}=K .
$$

According to (i), we find that $d \geq K$.

(iii) Consider the minimize sequence $\left\{u_{k}\right\}_{k}^{\infty} \subset u \in \aleph$ for $J$, verify

$$
\lim _{k \rightarrow \infty} J\left(u_{k}\right)=d
$$

Hence, we have $\left\{\left|u_{k}\right|\right\}_{k}^{\infty} \subset u \in \aleph$ is also a minimizing sequence for $J$ due to $\left|u_{k}\right| \subset u \in \aleph$ and $J\left(\left|u_{k}\right|\right)=J\left(u_{k}\right)$. For this, we can suppose that $u_{k}>0$ a.e. $\Omega$ for any $k \in u \in \aleph$.

From it, we note that $J$ is coercive on $u \in \aleph$; in other words, $\left\{u_{k}\right\}_{k}^{\infty}$ is bounded in $W_{0}^{1, p}(\Omega)$. Since $W_{0}^{1, p}(\Omega)^{\circ} L^{p}(\Omega$ ) is compact embedding, $\exists u$ is a function and a subsequence of $\left\{u_{k}\right\}_{k}^{\infty}$, still given by $\left\{u_{k}\right\}_{k}^{\infty}$, such that

$$
\begin{gathered}
u_{k} \rightarrow u \text { weakly in } W_{0}^{1, p}(\Omega), \\
u_{k} \rightarrow u \text { strongly in } L^{p}(\Omega), \\
u_{k}(x) \rightarrow u(x) \text { a.e.in } \Omega .
\end{gathered}
$$

Hence, $u \geq 0$ on $\Omega$ and

$$
\begin{aligned}
J(t) & =\frac{1}{p}\|\nabla u\|_{p}^{p}+\frac{p+1}{p^{2}}\|u\|_{p}^{p}-\frac{1}{p} \int_{\Omega} \ln |u| u^{p} d x \\
& \leq \lim _{k \rightarrow \infty} \inf \left(\frac{p+1}{p^{2}}\|u\|_{p}^{p}+\frac{1}{p}\|\nabla u\|_{p}^{p}-\frac{1}{p} \int_{\Omega} \ln |u| u^{p} d x\right) \\
& =\lim _{k \rightarrow \infty} \inf J\left(u_{k}\right)=d .
\end{aligned}
$$

We apply Lebesgue dominated convergence theorem and weak lower semicontinuity.

As $u_{k} \in u \in \aleph$, we have $u_{k} \in W_{0}^{1, p}(\Omega) \backslash\{0\}$ and $I\left(u_{k}\right)$ which implies

$$
\left\|u_{k}\right\|_{p} \geq e^{\left(n+p^{2}\right) / p^{2}}\left(\frac{p^{2}}{n \mathscr{L}_{p}}\right)^{n / p^{2}}=l .
$$

According to Lemma 8 , we have $\|u\|_{p} \neq 0$ converge strongly in $L^{p}(\Omega)$; that is to say, that $u \in W_{0}^{1, p}(\Omega) \backslash\{0\}$. Moreover, using weak lower continuity, we find

$$
\begin{aligned}
I(u) & =\|u\|_{p}^{p}+\|\nabla u\|_{p}^{p}-\int_{\Omega} \ln |u| u^{p} d x \\
& \leq \lim _{k \rightarrow \infty} \inf \left(\|u\|_{p}^{p}+\|\nabla u\|_{p}^{p}-\int_{\Omega} \ln |u| u^{p} d x\right) \\
& =\lim _{k \rightarrow \infty} \inf I\left(u_{k}\right)=0 .
\end{aligned}
$$

As a final stage of proof (iii), we prove that $I(u)=0$. If this is false, we get $I(u)<0$; hence, by Lemma $7, \exists \lambda^{*}<1$ which verifying $I\left(\lambda^{*} u\right)=0$. Further, we find

$$
\begin{aligned}
0<d \leq J\left(\lambda^{*} u\right) & =\frac{1}{p^{2}}\left\|\lambda^{*} u\right\|_{p}^{p} \leq \frac{\left(\lambda^{*}\right)^{p}}{p^{2}} \lim _{k \rightarrow \infty} \inf \left\|u_{k}\right\|_{p}^{p} \\
& =\left(\lambda^{*}\right)^{p} \lim _{k \rightarrow \infty} \inf J\left(u_{k}\right)=\left(\lambda^{*}\right)^{p} d<d .
\end{aligned}
$$

And it produces a stark contrast. Meaning that the proof of Lemma 9 has ended.

Definition 10. We say that function $u(t)$ represents a weak solution to problem (1) on $\Omega \times[0, T)$, if

$$
\begin{gathered}
u \in C\left((0, T) ; W_{0}^{1, p}(\Omega)\right) \cap C^{1}\left((0, T) ; L^{2}(\Omega)\right), \\
u_{t} \in L^{\infty}\left((0, T) ; L^{2}(\Omega)\right)
\end{gathered}
$$

satisfies

$$
\left\{\begin{array}{l}
\int_{\Omega}|\nabla u|^{p-2} \nabla u \nabla w d x+\int_{\Omega}|u|^{p-2} u w(x) d x+\int_{\Omega} u_{t} w(x) d x=k \int_{\Omega} \ln |u(x, t)| u^{p-2}(x, t) w(x) d x, \forall w \in H_{0}^{1}(\Omega) \\
u(x, 0)=u_{0}(x)
\end{array}\right.
$$

Lemma 11. Let $u_{0} \in W_{0}^{1, p}(\Omega) \backslash\{0\}$ and $l=e^{\left(n+p^{2}\right) / p^{2}}$ $\left(p^{2} / n \mathscr{L}_{p}\right)^{n / p^{2}}$. Suppose that $0<E(0)<l^{p} / p^{2}<d$.

(i) If $u_{0} \in W$, then $u \in W$ for $0 \leq t \leq T$

(ii) If $u_{0} \in V$, then $u \in V$ for $0 \leq t \leq T$, such that $T$ is the maximum time of existence of $u(t)$.

Proof.

(i) We put $T$ is the maximum time of existence of solution $u$. From (24) combined with (47), we find 


$$
J(u) \leq J\left(u_{0}\right)<d, \forall t \in[0, T) .
$$

Then, we have $u(t) \in W$ for every $t \in 0, T)$. If it is false, hence $\exists t_{0} \in[0, T)$ verify $u\left(t_{0}\right) \in \partial W$, we get either $I\left(u_{0}\right)=0$ and $\left\|\Delta\left(u_{0}\right)\right\| \neq 0$ or (b) $J\left(u_{0}\right)=d$.

According to (64), (b) is impossible, that is, $I\left(u_{0}\right)=0$ and $\left\|\Delta\left(u_{0}\right)\right\| \neq 0$. But it is $\exists J\left(u_{0}\right) \geq d$ if $0<d=\inf _{u \in \mathcal{N}} J(u)$. From this, we have a stark contrast, $u(t) \in W$ is obtained for $\forall t \in 0, T)$.

(ii) In the same way, we prove case (ii)

Theorem 12. Consider $u_{0}(x) \in W_{0}^{1, p}(\Omega) \backslash\{0\}$. If $I\left(u_{0}\right)>0$ and $E(0)<d$ or $\left\|u_{0}\right\|_{p}^{p}=0$. Therefore, problem (1) admits a weak global solution $u(t) \in L^{\infty}\left(0, \infty ; W_{0}^{1, p}(\Omega) \backslash\{0\}\right)$, $u_{t}(t) \in L^{\infty}\left(0, \infty ; L^{2}(\Omega)\right)$.
Proof. Consider the orthogonal basis $\left\{w_{j}\right\}_{j=1}^{\infty}$ of the "separable" space $W_{0}^{1, p}(\Omega)$ which is orthonormal in $L^{2}(\Omega)$. Let the following subspace $V_{m}$ on the finite dimensional

$$
V_{m}=\operatorname{span}\left\{w_{1}, w_{2}, \cdots, w_{m}\right\},
$$

where the projections of the initial data be defined by

$$
u_{0}^{m}(x)=\sum_{j=1}^{m} a_{j} w_{j}(x) \rightarrow u_{0} \text { in } H_{0}^{2}(\Omega),
$$

for all $j=1,2, \cdots, m$.

Now, we can see the approximated solutions of (1) as in the following form

$$
u^{m}(x, t)=\sum_{j=1}^{m} h_{j}^{m}(t) w_{j}(x)
$$

of the approximate problem in $V_{m}$

$$
\left\{\begin{array}{l}
\int_{\Omega}\left|\nabla u^{m}\right|^{p-2} \nabla u^{m} \nabla w d x+\int_{\Omega}\left|u^{m}\right|^{p-2} u^{m} w(x) d x+\int_{\Omega} u_{t}^{m} w(x) d x=k \int_{\Omega}\left|u^{m}\right|^{p-2}(x, t) \ln \left|u^{m}(x, t)\right| w(x) d x, w \in V_{m}, \\
u^{m}(0)=u_{0}^{m}=\sum_{j=1}^{m}\left(u_{0}, w_{j}\right) w_{j} .
\end{array}\right.
$$

It produces an ordinary differential equation system (ODE) made up of unknown functions $h_{j}^{m}(t)$. Starting from the standard theory of existence, there are functions

$$
h_{j}:\left[0, t_{m}\right) \rightarrow R, j=1,2, \cdots, m,
$$

which verify (68) in a maximal interval $\left[0, t_{m}\right), 0<t_{m} \leq T$. Next, we prove that $t_{m}=T$ and that the local solution is uniformly bounded independent of $m$ and $t$. For this purpose, let us replace $w$ by $u_{t}^{m}$ in (68) and integrate by parts, we get

$$
\frac{d}{d t} E^{m}(t)=-\left\|u_{t}^{m}\right\|^{2} \leq 0
$$

such as

$$
E^{m}(t)=\frac{1}{p}\left\|\nabla u^{m}\right\|_{p}^{p}+\frac{p+1}{p^{2}}\left\|u^{m}\right\|_{p}^{p}-\frac{1}{p} \int_{\Omega}\left|u^{m}\right|^{p} \ln \left|u^{m}\right| d x .
$$

Integrating (70) from 0 to $t$, and using (24), we obtain

$$
J\left(u^{m}\right)+\int_{0}^{t}\left\|u_{s}^{m}\right\|^{2} d s=E^{m}(0) .
$$

According to (68), with $m \rightarrow \infty$, we find $E^{m}(0) \rightarrow E(0)$. We select $m$ large enough; we find

$$
J\left(u^{m}\right)+\int_{0}^{t}\left\|u_{s}^{m}\right\|^{2} d s<d
$$

Hence, by (23), we have

$$
J(u)=\frac{1}{p} I(u)+\frac{1}{p^{2}}\|u\|_{p}^{p} .
$$

By $u_{0} \in W$,

$$
J\left(u^{m}(0)\right)=E(0) ;
$$

we select $m$ large enough and $0 \leq t<\infty$; we find $u^{m}(0) \in W$. By (24) and Lemma 11, by picking $m$ large enough and $0 \leq$ $t<\infty$, we get ${ }^{m}(t) \in W$. Further, according to (24) and (21), we obtain

$$
\frac{1}{p}\left\|\nabla u^{m}\right\|_{p}^{p}+\frac{p+1}{p^{2}}\left\|u^{m}\right\|_{p}^{p}-\frac{1}{p} \int_{\Omega}\left|u^{m}\right|^{p} \ln \left|u^{m}\right| d x+\int_{0}^{t}\left\|u_{s}^{m}\right\|^{2} d s<d,
$$


where $0 \leq t<\infty$. By choosing $m$ large enough and $0 \leq t<\infty$ (76), we get

$$
\begin{aligned}
\left\|\nabla u^{m}\right\|_{p}^{p} & <p d, \\
\left\|u^{m}\right\|_{p}^{p} & <\frac{p^{2}}{p+1} d, \\
\int_{0}^{t}\left\|u_{s}^{m}\right\|^{2} d s & <d .
\end{aligned}
$$

According to Remark 5, we find

$$
\begin{aligned}
\int_{\Omega}\left|u^{m}\right|^{p} \ln \left|u^{m}\right| d x \leq & \int_{\Omega_{1}}\left|u^{m}\right|^{p} \ln \left|u^{m}\right| d x \\
& +\int_{\Omega_{2}}\left|u^{m}\right|^{p} \ln \left|u^{m}\right| d x \\
\leq & C \int_{\Omega_{2}}\left|u^{m}\right|^{p+\zeta} d x \leq C\left\|u^{m}\right\|_{p+\zeta}^{p+\zeta},
\end{aligned}
$$

where $\zeta$ is pick satisfying $p+\zeta<n p /(n-p)$ as $p<n$ and $\zeta>0$ as $p \geq n$ and $\Omega_{1}=\left\{x \in \Omega:\left|u^{m}(x)<1\right|\right\}$ and $\Omega_{2}=\{x \in \Omega: \mid$ $\left.u^{m}(x) \geq 1 \mid\right\}$.

Applying the embedding theorem, Lemma 6 and Young's inequality, gives from (78):

$$
\begin{aligned}
\int_{\Omega} \ln & \left|u^{m} \| u^{m}\right|^{p} d x \\
& \leq C\left\|u^{m}\right\|_{p+\zeta}^{p+\zeta} \\
& \leq C\left\|\nabla u^{m} u\right\|_{p}^{\alpha(p+\zeta)}\left\|u^{m}\right\|_{p}^{(1-\alpha)(p+\zeta)} \\
& \leq \varepsilon\left\|\nabla u^{m} u\right\|_{p}^{p}+C_{\varepsilon}\left(\left\|u^{m}\right\|_{p}^{p}\right)^{(1-\alpha) p(p+\zeta) / p[p-\alpha(p+\zeta)]} \\
& \leq C_{\varepsilon}\left\|\nabla u^{m} u\right\|_{p}^{p} .
\end{aligned}
$$

Therefore, we choose $0<\zeta$ for $p>\alpha(p+\zeta)$, where $\varepsilon \in(0$ ,1) with

$$
\alpha=\left(\frac{1}{p}-\frac{1}{p+\zeta}\right)\left(\frac{1}{n}-\frac{1}{p}+\frac{1}{p}\right)^{-1}, \frac{(1-\alpha) p(p+\zeta)}{p-\alpha(p+\zeta)}>1 .
$$

Using (79) and (76), for $0 \leq t<\infty$, we find

$$
\int_{\Omega} \ln \left|u^{m}\right|\left|u^{m}\right|^{p} d x<C_{\varepsilon} p d
$$

Hence, we get

$\left\{\begin{array}{l}u^{m} \text { is uniformly bounded in } L^{\infty}\left(0, \infty ; W_{0}^{1, p}(\Omega)\right), \\ u_{t}^{m} \text { is uniformly bounded in } L^{\infty}\left(0, \infty ; L^{2}(\Omega)\right) .\end{array}\right.$
Using the integration on (68), we get for $0 \leq t<\infty$

$$
\begin{aligned}
\int_{\Omega} u^{m} w_{s} d x= & \int_{\Omega} u_{0} w_{s} d x+\int_{0}^{t} \int_{\Omega} \ln \left|u^{m}\right|^{k}\left|u^{m}\right|^{p-1} w_{s} d x d s \\
& -\int_{0}^{t} \int_{\Omega}\left|\nabla u^{m}\right|^{p-2}(s) \nabla u^{m}(s) \nabla w_{s} d x d s \\
& -\int_{0}^{t} \int_{\Omega}\left|u^{m}\right|^{p-2}(s) u^{m}(s) w_{s} d x .
\end{aligned}
$$

Further, after passing through the limit in (ref 4030), we arrive at the weak solution left $(u$ right) to the problem (ref $300)$. According to the initial data in (ref 300), we conclude that $(u(x, 0))=\left(u_{0}\right)$ in $W_{0}^{1, p}$.

\section{Decay of Solution}

In this section, by using the Lyapunov functional, we show the decay of solution to (1).

First, we define the Lyapunov functional by

$$
L(t)=E(t)+\frac{\varepsilon}{2} \int_{\Omega} u^{2} d x
$$

where $\varepsilon>0$. We will prove the equivalence between $L(t)$ and $E(t)$.

Lemma 13. For $\varepsilon>0$ small enough, we have

$$
\beta_{1} L(t) \leq E(t) \leq \beta_{2} L(t),
$$

where $\beta_{1}, \beta_{2}>0$.

We find $L \sim E$ by choosing $\varepsilon$ small enough.

Theorem 14. Let $u_{0} \in V$. Assume further $0<E(0)<(p+1) /$ $p^{2} \mu l^{p}<d$, where

$$
\begin{gathered}
l=e^{n+p^{2} / p^{2}}\left(\frac{p^{2}}{n \mathscr{L}_{p}}\right)^{n / p^{2}}, \\
\mu^{n-p^{2} / n p} e^{(1-p)\left(n+p^{2}\right)}\left(\frac{p}{n \mathscr{L}_{p}}\right)^{1-p / p}<\mu<\frac{p(\beta-p)+\beta C^{*}}{(\beta-p)} ;
\end{gathered}
$$

hence, $\exists c_{1}, c_{2}>0$ satisfies

$$
0<E(t) \leq c_{1} e^{-c_{2} t}, t \geq 0 .
$$

Proof. A differentiation of $L(t)$ and equation (1) gives

$$
\begin{aligned}
L^{\prime}(t)= & E^{\prime}(t)+\varepsilon \int_{\Omega} u u_{t} d x=-\left\|u_{t}\right\|^{2} \\
& -\varepsilon\left(\|\nabla u\|_{p}^{p}+\|u\|_{p}^{p}\right)+\varepsilon \int_{\Omega} \ln |u| u^{p} d x .
\end{aligned}
$$


Adding and subtracting $\varepsilon \beta E(t)$ into (88) $(\beta>0)$, we obtain

$$
\begin{aligned}
L^{\prime}(t)= & -\left\|u_{t}\right\|^{2}+\varepsilon\left(\frac{\beta-p}{p}\right)\|\nabla u\|_{p}^{p}+\varepsilon\left(\frac{\beta-p}{p}\right)\|u\|_{p}^{p} \\
& +\varepsilon\left(1-\frac{\beta}{p}\right) \int_{\Omega} \ln |u| u^{p} d x+\frac{1}{p^{2}} \varepsilon \beta\|u\|_{p}^{p}-\varepsilon \beta E(t) \\
\leq & -\left\|u_{t}\right\|^{2}+\varepsilon\left(\frac{\beta-p}{p}\right)\left(1+\frac{C^{*} \beta}{p(\beta-p)}\right)\|\nabla u\|_{p}^{p} \\
& +\varepsilon\left(\frac{\beta-p}{p}\right)\|u\|_{p}^{p}+\varepsilon\left(1-\frac{\beta}{p}\right) \int_{\Omega} \ln |u| u^{p} d x .
\end{aligned}
$$

Using the inequality of logarithmic Sobolev together with $\|u\|_{p}^{p} \leq C^{*}\|\nabla u\|_{p}^{p}\left(C^{*}>0\right)$ gives

$$
\begin{aligned}
L^{\prime}(t) \leq & -\left\|u_{t}\right\|^{2}+\varepsilon\left(\frac{\beta-p}{p}\right)\left(1+\frac{C^{*} \beta}{p(\beta-p)}\right)\|\nabla u\|_{p}^{p} \\
& +\varepsilon\left(\frac{\beta-p}{p}\right)\|u\|_{p}^{p}+\varepsilon\left(1-\frac{\beta}{p}\right) \int_{\Omega} \ln |u| u^{p} d x-\varepsilon \beta E(t) \\
\leq & -\varepsilon \beta E(t)-\left\|u_{t}\right\|^{2}+\varepsilon\left(\frac{\beta-p}{p}\right)\left(1+\frac{C^{*} \beta}{p(\beta-p)}-\frac{\mu}{p}\right)\|\nabla u\|_{p}^{p} \\
& -\varepsilon\left(\frac{\beta-p}{p}\right)\left[\ln \|u\|_{p}-\left(\frac{n}{p^{2}} \ln \left(\frac{p \mu e}{n \mathscr{L}_{p}}\right)+1\right)\right]\|u\|_{p}^{p} .
\end{aligned}
$$
find

Noting that $0<\beta<p$ and using (21) and Theorem 12, we

$$
\begin{aligned}
\ln \|u\|_{p} & \leq \ln \left(\frac{p^{2}}{p+1} J(u)\right) \leq \ln \left(\frac{p^{2}}{p+1} E(t)\right) \\
& \leq \ln \left(\frac{p^{2}}{p+1} E(0)\right) \leq \ln \left(\mu l^{p}\right) \\
& =\ln \left(\mu e^{n+p^{2} / p}\left(\frac{p \mu}{n \mathscr{L}_{p}}\right)^{n / p}\right) .
\end{aligned}
$$

By $\mu$ satisfying

$$
\mu^{n-p^{2} / n p} e^{(1-p)\left(n+p^{2}\right)}\left(\frac{p}{n \mathscr{L}_{p}}\right)^{1-p / p}<\mu<\frac{p(\beta-p)+\beta C^{*}}{(\beta-p)},
$$

we guarantee

$$
\begin{gathered}
\left(1+\frac{C^{*} \beta}{p(\beta-p)}-\frac{\mu}{p}\right)>0, \\
\ln \|u\|_{p}-\left(\frac{n}{p^{2}} \ln \left(\frac{p \mu e}{n \mathscr{L}_{p}}\right)+1\right)>0 ;
\end{gathered}
$$

then, we obtain

$$
L^{\prime}(t) \leq-\varepsilon \beta E(t)-\left\|u_{t}\right\|^{2} .
$$

Hence, inequality (95) becomes

$$
L^{\prime}(t) \leq-\varepsilon \beta E(t) .
$$

According to (85), we get

$$
L^{\prime}(t) \leq-\varepsilon \beta \beta_{2} L(t) .
$$

Setting $c_{2}=\varepsilon \beta \beta_{2}>0$ and integrating (97) yield

$$
L(t) \leq c_{1} e^{-c_{2} t} .
$$

Finally, by (85), we obtain (87). This is the end of the proof.

\section{Conclusion}

As mentioned earlier in the introduction, the majority of problems in science are nonlinear and their analytical solutions are not easy to find, and most physical problems mostly use higher nonlinear partial differential equations (PDEs). It has been found to be extremely difficult to find accurate or analytical solutions to such problems. However, in the past several centuries, many scientists have made great progress and adopted various techniques to study the analytical side of these famous problems, and nonlinear logarithmic has also received much attention from physicists and mathematicians. Log nonlinearity was introduced into the relativistic wave equation describing spinning particles moving in an external electromagnetic field and in the relativistic wave equation (see, for example, [1-3, 6, 14, 18, 19, 29, 36, 37]); in this contribution, under some sufficient initial and boundary conditions, we have studied the analytical side of $p$ -Laplacian heat equations with respect to logarithmic nonlinearity in the right-hand side, where the global existence and decay estimates of weak solutions are proved. In the next work, we extend our recent work to the coupled system for this important problem. Also, some numerical examples will be given in order to ensure the theory study by using some famous algorithms which are presented in $[38,39]$.

\section{Data Availability}

No data were used to support the study.

\section{Conflicts of Interest}

The authors declare that they have no conflicts of interest.

\section{Acknowledgments}

The fourth author extends their appreciation to the Deanship of Scientific Research at King Khalid University for funding this work through a research group program under grant (R.G.P-2/1/42). 


\section{References}

[1] C. O. Alves and T. Boudjeriou, "Existence of solution for a class of heat equation involving the $\mathrm{p}(\mathrm{x})$ Laplacian with triple regime," Zeitschrift für angewandte Mathematik und Physik, vol. 72, no. 1, 2021.

[2] H. Buljan, A. Siber, M. Soljacic, T. Schwartz, M. Segev, and D. N. Christodoulides, "Incoherent white light solitons in logarithmically saturable noninstantaneous nonlinear media," Physics Review, vol. 68, pp. 258-275, 2003.

[3] M. M. Chaharlang, M. A. Ragusa, and A. Razani, "A sequence of radially symmetric weak solutions for some nonlocal elliptic problem in R - N," Mediterranean Journal of Mathematics, vol. 17 , no. $2,2020$.

[4] P. Chen and M. Gurtin, "On a theory of heat conduction involving two temperatures," Zeitschrift für Angewandte Mathematik und Physik, vol. 19, no. 4, pp. 614-627, 1968.

[5] M. Del Pino and J. Dolbeault, "Diffusions non lineaires et constantes optimales dans des inegalites de type Sobolev : comportement asymptotique d'equations faisant intervenir le $p$ -Laplacien," Comptes Rendus Mathematique, vol. 334, no. 5, pp. 365-370, 2002.

[6] M. A. Goodrich and M. A. Ragusa, "Holder continuity of weak solutions of p-Laplacian PDEs with VMO coefficients," Nonlinear Analysis-Theory Methods and Applications, vol. 185, pp. 336-355, 2019.

[7] K. Enqvist and J. McDonald, "Q-balls and baryogenesis in the MSSM,” Physics Letters B, vol. 425, no. 3-4, pp. 309-321, 1998.

[8] T. Hiramatsu, M. Kawasaki, and F. Takahashi, "Numerical study of Q-ball formation in gravity mediation," Journal of Cosmology and Astroparticle Physics, vol. 2010, no. 6, 2010.

[9] K. Hosseini, M. Mirzazadeh, F. Rabiei, H. M. Baskonus, and G. Yel, "Dark optical solitons to the Biswas-Arshed equation with high order dispersions and absence of the self-phase modulation," Optik, vol. 209, article 164576, 2020.

[10] K. Hosseini, M. Samavat, M. Mirzazadeh, W. X. Ma, and Z. Hammouch, "A new (3+1)-dimensional Hirota bilinear equation: its Bäcklund transformation and rational-type solutions," Regular and Chaotic Dynamics, vol. 25, no. 4, pp. 383391, 2020.

[11] B. Hu and H.-M. Yin, "Semilinear parabolic equations with prescribed energy," Rendiconti del Circolo Matematico di Palermo, vol. 44, no. 3, pp. 479-505, 1995.

[12] S. Boularas, "Solvability of the Moore-Gibson-Thompson equation with viscoelastic memory term and integral condition via Galerkin method," Fractals, 2021.

[13] N. Ioku, "The Cauchy problem for heat equations with exponential nonlinearity," Journal of Differential Equations, vol. 251, no. 4-5, pp. 1172-1194, 2011.

[14] E. Piskin, S. Boulaaras, and N. Irkil, "Qualitative analysis of solutions for thep-Laplacian hyperbolic equation with logarithmic nonlinearity," Mathematicsl Methods in the Applied Sciences, vol. 44, no. 6, pp. 4654-4672, 2021.

[15] C. Qu, X. Bai, and S. Zheng, "Blow-up versus extinction in a nonlocal $p$-Laplace equation with Neumann boundary conditions," Journal of Mathematical Analysis and Applications, vol. 412, no. 1, pp. 326-333, 2014.

[16] E. Piskin and N. Irkil, "Mathematical behavior of solutions of p-Laplacian equation with logarithmic source term," Sigma Journal of Engineering and Natural Sciences, vol. 10, pp. 213220, 2019.
[17] V. S. Vladimirov, "The equation of the p-adic open string for the scalar tachyon field," Zvestiya: Mathematics, vol. 69, pp. 487-512, 2005.

[18] I. Bialynicki-Birula and J. Mycielski, "Nonlinear wave mechanics," Annals of Physics, vol. 100, no. 1-2, pp. 62-93, 1976.

[19] P. Gorka, "Logarithmic Klein-Gordon equation," Acta Physica Polonica B, vol. 40, pp. 59-66, 2009.

[20] I. Bialynicki-Birula and J. Mycielski, "Wave equations with logarithmic nonlinearities," Bulletin de l'Academie Polonaise des Sciences. Serie des Sciences, Mathematiques, Astronomiques et Physiques, vol. 23, pp. 461-466, 1975.

[21] S. Boulaaras, A. Draifia, and M. Alnegga, "Polynomial decay rate for Kirchhoff type in viscoelasticity with logarithmic nonlinearity and not necassarily decreasing kernel," Symmetry, vol. 11, pp. 1-24, 2019.

[22] T. Cazenave and A. Haraux, "Équations d'évolution avec non linéarité logarithmique," Annales de la faculté des sciences de Toulouse Mathématiques, vol. 2, no. 1, pp. 21-51, 1980.

[23] H. Chen, P. Luo, and G. Liu, "Global solution and blow-up of a semilinear heat equation with logarithmic nonlinearity," Journal of Mathematical Analysis and Applications, vol. 422, no. 1, pp. 84-98, 2015.

[24] H. Chen and S. Y. Tian, "Initial boundary value problem for a class of semilinear pseudo-parabolic equations with logarithmic nonlinearity," Journal of Differential Equations, vol. 258, pp. 84-98, 2015.

[25] Y. Cao and C. Liu, "Initial boundary value problem for a mixed pseudo-parabolic p-Laplacian type equation with logarithmic nonlinerity," Electronic Journal of Differential Equations, vol. 116, pp. 1-19, 2018.

[26] X. S. Han, "Global existence of weak solutions for a logarithmic wave equation arising from Q-ball dynamics," Bulletin of the Korean Mathematical Society, vol. 50, no. 1, pp. 275-283, 2013.

[27] C. N. Le and X. T. Le, "Global solution and blow up for a class of pseudo p-Laplacian evolution equations with logarithmic nonlinearity," Computers \& Mathematcs with Applications, vol. 73, pp. 2076-2091, 2017.

[28] A. Merah, F. Mesloub, S. M. Boulaaras, and B.-B. Cherif, "A new result for a blow-up of solutions to a logarithmic flexible structure with second sound," Advances in Mathematical Physics, vol. 2021, Article ID 5555930, 7 pages, 2021.

[29] A. Choucha, S. Boulaaras, D. Ouchenane, and S. Beloul, "General decay of nonlinear viscoelastic Kirchhoff equation with Balakrishnan-Taylor damping, logarithmic nonlinearity and distributed delay terms," Mathematicsl Methods in the Applied Sciences, vol. 2020, 2020.

[30] L. Yan and Z. Yang, "Blow-up and non-extinction for a nonlocal parabolic equation with logarithmic nonlinearity," Boundary Value Problems, vol. 2018, no. 1, 2018.

[31] H. W. Zhang, G. W. Liu, and Q. Y. Hu, "Asymptotic behavior for a class of logarithmic wave equations with linear damping," Applied Mathematics and Optimization, vol. 79, pp. 131-144, 2019.

[32] Y. Wu and X. Xue, "Uniform decay rate estimates for a class of quasilinear hyperbolic equations with nonlinear damping and source terms," Applicable Analysis, vol. 92, no. 6, pp. 11691178, 2013.

[33] E. Piskin, "On the decay and blow up of solutions for a quasilinear hyperbolic equations with nonlinear damping and source terms," Boundary Value Problems, vol. 127, 2015. 
[34] O. A. Ladyzhenskaya, V. A. Solonnikov, and N. N. Uralyseva, "Linear and quasi-linear equations of parablic type," in Transl. Matah. Monogr, Mauka Moskow, vol. 23, American Mathematical Soc., 1967.

[35] L. E. Payne and D. H. Sattinger, "Saddle points and instability of nonlinear hyperbolic equations," Israel Journal of Mathematics, vol. 22, no. 3-4, pp. 273-303, 1975.

[36] S. Boulaaras and Y. Bouizem, "Blow up of solutions for a nonlinear viscoelastic system with general source term," Quaestiones Mathematicae, pp. 1-11, 2020.

[37] S. Boulaaras, Y. Bouizem, and R. Guefaifia, "Further results of existence of positive solutions of elliptic Kirchhoff equation with general nonlinearity of source terms," Mathematicsl Methods in the Applied Sciences, vol. 43, no. 15, pp. 91959205, 2020.

[38] S. Boulaaras and M. Haiour, "The finite element approximation of evolutionary Hamilton-Jacobi-Bellman equations with nonlinear source terms," Indagationes Mathematicae, vol. 24, no. 1, pp. 161-173, 2013.

[39] S. Boulaaras and M. Haiour, “_L_ ${ }^{\infty}$-asymptotic behavior for a finite element approximation in parabolic quasi-variational inequalities related to impulse control problem," Applied Mathematics and Computation, vol. 217, no. 14, pp. 64436450, 2011. 